\title{
ON CERTAIN UNIVALENT CLASS ASSOCIATED WITH FIRST ORDER DIFFERENTIAL SUBORDINATIONS
}

\author{
RABHA W. IBRAHIM
}

\begin{abstract}
In this paper, we consider certain differential inequalities and first order differential subordinations. As their applications, we obtain some sufficient conditions for univalence, which generalize and refine some previous results.
\end{abstract}

\section{Introduction}

Let $\mathscr{H}$ be the class of functions analytic in the unit disk $U=\{z:|z|<1\}$ and for $a \in \mathbb{C}$ (set of complex numbers) and $n \in \mathbb{N}$ (set of natural numbers), let $\mathscr{H}[a, n]$ be the subclass of $\mathscr{H}$ consisting of functions of the form $f(z)=a+a_{n} z^{n}+a_{n+1} z^{n+1}+\cdots$. Let $\mathscr{A}$ be the class of functions $f$, analytic in $U$ and normalized by the conditions $f(0)=f^{\prime}(0)-1=0$.

Let $f$ be analytic in $U$, g analytic and univalent in $U$ and $f(0)=g(0)$. Then, by the symbol $f(z)<g(z)$ (f subordinate to $g$ ) in $U$, we shall mean $f(U) \subset g(U)$.

Let $\phi: \mathbb{C}^{2} \rightarrow \mathbb{C}$ and let $h$ be univalent in $U$. If $p$ is analytic in $U$ and satisfies the differential subordination $\left.\phi(p(z)), z p^{\prime}(z)\right)<h(z)$ then $p$ is called a solution of the differential subordination. The univalent function $q$ is called a dominant of the solutions of the differential subordination, $p<q$. If $p$ and $\left.\phi(p(z)), z p^{\prime}(z)\right)$ are univalent in $U$ and satisfy the differential superordination $\left.h(z) \prec \phi(p(z)), z p^{\prime}(z)\right)$ then $p$ is called a solution of the differential superordination. An analytic function $q$ is called subordinant of the solution of the differential superordination if $q \prec p$.

The function $f \in \mathscr{A}$ is called $\Phi$-like if

$$
\Re\left\{\frac{z f^{\prime}(z)}{\Phi(f(z))}\right\}>0, z \in U .
$$

This concept was introduced by Brickman [1] and established that a function $f \in \mathscr{A}$ is univalent if and only if $f$ is $\Phi$-like for some $\Phi$.

2010 Mathematics Subject Classification. 30C45.

Key words and phrases. Univalent functions, starlike functions, convex functions, close-to-convex functions, differential subordination, subordination, superordination, unit disk, $\Phi$-like functions.. 
Definition 1.1. Let $\Phi$ be analytic function in a domain containing $f(U), \Phi(0)=0, \Phi^{\prime}(0)=1$ and $\Phi(\omega) \neq 0$ for $\omega \in f(U)-0$. Let $q(z)$ be a fixed analytic function in $U, q(0)=1$. The function $f \in \mathscr{A}$ is called $\Phi$-like with respect to $q$ if

$$
\frac{z f^{\prime}(z)}{\Phi(f(z))} \prec q(z), z \in U
$$

Ruscheweyh [2] investigated this general class of $\Phi$-like functions.

In the present paper, we consider another new class $H\left(\alpha, \lambda ; \Phi_{1}(f(z)), \Phi_{2}(f(z))\right)$ involving two different types of $\Phi$-like functions, $\Phi_{1}$ and $\Phi_{2}$, which defined by

$$
\frac{z f^{\prime}(z)}{\Phi_{1}(f(z))}\left\{(1-\alpha) \frac{z f^{\prime}(z)}{\Phi_{2}(f(z))}+\alpha\left(1+\frac{\lambda z f^{\prime \prime}(z)}{f^{\prime}(z)}\right)\right\}<F(z),
$$

where $\alpha \in[0,1], \lambda \in \mathbb{R}, F$ is the conformal mapping of the unit disk $U$ with $F(0)=1$ and $\Phi_{1}$ and $\Phi_{2}$ satisfy Definition 1.1.

Remark 1. As special cases of the class $H\left(\alpha, \lambda ; \Phi_{1}(f(z)), \Phi_{2}(f(z))\right)$ are the following well known classes: $H\left(0 ; \Phi(f(z)), z f^{\prime}(z)\right)$ (see [2]); $H\left(\alpha, 1 ; z f^{\prime}(z), z\right)$ (see [3-5]); $H(1, \lambda ; f(z))$ (see [6-16]). Also this class reduces to the classes of starlike functions, convex functions and close-toconvex functions.

In order to obtain our results, we need the following lemmas.

Lemma 1.([17]) Let $w(z)$ be analytic in $U$ with $w(0)=0$. If $|w(z)|$ attains its maximum value on the circle $|z|=r<1$ at a point $z_{0}$, then

$$
z_{0} w^{\prime}\left(z_{0}\right)=k w\left(z_{0}\right)
$$

where $k$ is a real number and $k \geq 1$.

Lemma 2.([18]) Let $q(z)$ be univalent in the unit disk $U$ and $\theta$ and $\phi$ be analytic in a domain $D$ containing $q(U)$ with $\phi(w) \neq 0$ when $w \in q(U)$. Set $Q(z):=z q^{\prime}(z) \phi(q(z)), h(z):=\theta(q(z))+$ $Q(z)$. Suppose that

1. $Q(z)$ is starlike univalent in $U$, and

2. $\Re\left\{\frac{z h^{\prime}(z)}{Q(z)}\right\}>0$ for $z \in U$.

If $\theta(p(z))+z p^{\prime}(z) \phi(p(z))<\theta(q(z))+z q^{\prime}(z) \phi(q(z))$ then $p(z) \prec q(z)$ and $q(z)$ is the best dominant.

2. The class $H\left(\alpha, \lambda ; \Phi_{1}(f(z)), \Phi_{2}(f(z))\right)$ 
Let us consider the sufficient condition for $f(z) \in \mathscr{A}$ to be in $H\left(\alpha, \lambda ; \Phi_{1}(f(z)), \Phi_{2}(f(z))\right)$. Our first result is contained in

Theorem 1. Let $p_{1}(z):=\frac{z f^{\prime}(z)}{\Phi_{1}(f(z))}$ and $p_{2}=\frac{z f^{\prime}(z)}{\Phi_{2}(f(z))}$. If $f \in \mathscr{A}$ satisfies

$$
\begin{aligned}
\Re\left\{(1-\alpha) z\left(p_{1}(z) p_{2}(z)\right)^{\prime}\right. & +\alpha z\left[p_{1}^{\prime}(z)+\lambda\left(\frac{z f^{\prime \prime}(z)+p_{2}^{\prime}(z) \Phi_{2}^{\prime}(z)+p_{2}^{\prime \prime}(z) \Phi_{2}(z)}{f^{\prime}(z)}\right.\right. \\
& \left.\left.\left.-\frac{p_{2}^{\prime}(z) \Phi_{2}(z) f^{\prime \prime}(z)}{\left(f^{\prime}(z)\right)^{2}}\right)\right]\right\} \\
& <\frac{2 \alpha}{(1-\alpha)^{2}},(z \in U)
\end{aligned}
$$

for some $\alpha \neq 1, \lambda \in \mathbb{R}$ then $f \in H\left(\alpha, \lambda ; \Phi_{1}(f(z)), \Phi_{2}(f(z))\right)$.

Proof Let $w(z)$ defined by

$$
H(z):=\frac{z f^{\prime}(z)}{\Phi_{1}(f(z))}\left\{(1-\alpha) \frac{z f^{\prime}(z)}{\Phi_{2}(f(z))}+\alpha\left(1+\frac{\lambda z f^{\prime \prime}(z)}{f^{\prime}(z)}\right)\right\}=\frac{\alpha+w(z)}{\alpha-w(z)}, \quad(\alpha \neq w(z)) .
$$

Then

$$
w(z)=\frac{\alpha(H(z)-1)}{1+H(z)}
$$

is analytic in $U$ with $w(0)=0$. It follows that

$$
\Re\left\{z H^{\prime}(z)\right\}=\Re\left\{\frac{2 \alpha z w^{\prime}(z)}{(\alpha-w(z))^{2}}\right\}<\frac{2 \alpha}{(1-\alpha)^{2}}, \alpha \neq 1 .
$$

Now we proceed to prove that $|w(z)|<1$. Suppose that there exists a point $z_{0} \in U$ such that

$$
\max _{|z| \leq\left|z_{0}\right|}|w(z)|=\left|w\left(z_{0}\right)\right|=1 .
$$

Then, using the Lemma 1 and letting $w\left(z_{0}\right)=e^{i \theta}$ and $z_{0} w^{\prime}\left(z_{0}\right)=k e^{i \theta}, k \geq 1$ yields

$$
\begin{aligned}
\Re\left\{z_{0} H^{\prime}\left(z_{0}\right)\right\} & =\Re\left\{\frac{2 \alpha z_{0} w^{\prime}\left(z_{0}\right)}{\left(\alpha-w\left(z_{0}\right)\right)^{2}}\right\} \\
& =\Re\left\{\frac{2 \alpha k e^{i \theta}}{\left(\alpha-e^{i \theta}\right)^{2}}\right\} \\
& \geq \frac{2 \alpha}{(1-\alpha)^{2}} .
\end{aligned}
$$

Thus we have

$$
\begin{aligned}
\Re\left\{z_{0} H^{\prime}\left(z_{0}\right)\right\} & =\Re\left\{(1-\alpha) z_{0}\left(p_{1}\left(z_{0}\right) p_{2}\left(z_{0}\right)\right)^{\prime}\right. \\
& +\alpha z_{0}\left[p_{1}^{\prime}\left(z_{0}\right)+\lambda\left(\frac{z_{0} f^{\prime \prime}\left(z_{0}\right)+p_{2}^{\prime}\left(z_{0}\right) \Phi_{2}^{\prime}\left(z_{0}\right)+p_{2}^{\prime \prime}\left(z_{0}\right) \Phi_{2}\left(z_{0}\right)}{f^{\prime}\left(z_{0}\right)}\right.\right. \\
& \left.\left.\left.-\frac{p_{2}^{\prime}\left(z_{0}\right) \Phi_{2}\left(z_{0}\right) f^{\prime \prime}\left(z_{0}\right)}{\left(f^{\prime}\left(z_{0}\right)\right)^{2}}\right)\right]\right\} \\
& \geq \frac{2 \alpha}{(1-\alpha)^{2}},(z \in U)
\end{aligned}
$$


which contradicts the hypothesis (3). Therefore, we conclude that $|w(z)|<1$ for all $z \in U$ that is $f \in H\left(\alpha, \lambda ; \Phi_{1}(f(z)), \Phi_{2}(f(z))\right)$. This completes the proof.

Corollary 1. If $f(z) \in \mathscr{A}$ satisfies the condition in Theorem 1 , then for $\alpha \in[0,1)$

$$
\left|H(z)-\frac{1+\alpha}{1-\alpha}\right|<\frac{1+\alpha}{1-\alpha} \text {. }
$$

Proof. Since $f \in H\left(\alpha, \lambda ; \Phi_{1}(f(z)), \Phi_{2}(f(z))\right)$ yields

$$
|w(z)|=\left|\frac{\alpha(H(z)-1)}{H(z)+1}\right|<1
$$

we obtain (6).

Next results show the starlikeness $\left(\mathscr{S}^{*}\right)$, convexity $(\mathscr{C})$ and close to convex $(\mathscr{K})$ for different order.

By letting $\alpha=0, \Phi_{1}(z)=f(z)$ and $\Phi_{2}(z)=z f^{\prime}(z)$ we have the following result

Corollary 2. If $f(z) \in \mathscr{A}$ satisfies the condition in Theorem 1 , then for $\alpha \in[0,1)$

$$
\left|\frac{z f^{\prime}(z)}{f(z)}-1\right|<1
$$

This implies that $f(z) \in \mathscr{S}^{*}$ and $\int_{0}^{z} \frac{f(t)}{t} d t \in \mathscr{C}$.

By setting $\alpha=0, \Phi_{1}(z)=g(z)$ where $g$ is starlike and satisfies $g(0)=0$ and $g^{\prime}(0)=1$ and $\Phi_{2}(z)=z f^{\prime}(z)$ we have the following result

Corollary 3. If $f(z) \in \mathscr{A}$ satisfies the condition in Theorem 1, then for $\alpha \in[0,1)$

$$
\left|\frac{z f^{\prime}(z)}{g(z)}-1\right|<1
$$

This implies that $f(z) \in \mathscr{K}$.

Note that Corollary 2 and Corollary 3 implies the univalence of the class $H\left(\alpha, \lambda ; \Phi_{1}(f(z)), \Phi_{2}(f(z))\right)$.

\section{The region of variability}

In this section, we show that for $\alpha \in[0,1)$ and $f \in H\left(\alpha, \lambda ; \Phi_{1}(f(z)), \Phi_{2}(f(z))\right)$ then $f$ is univalent in $U$. Moreover, we estimate the region of variability. We prove a subordination 
theorem by using Lemma 2 and as applications of this result, we find the sufficient conditions for $f \in \mathscr{A}$ to be univalent.

Theorem 2. Let $q, q(z) \neq 0$, be a univalent function in $U$, and $g(z) \neq 0$ be analytic in $\mathbb{C}$ such that for nonnegative real numbers $\mu$ and $v$

$$
\Re\left\{1+\frac{z q^{\prime \prime}(z)}{q^{\prime}(z)}-\frac{z q^{\prime}(z)}{q(z)}\right\}>\max \left\{0,\left(\frac{\mu}{v}\right) \Re\left(q(z)\left[1+\frac{g^{\prime}(z)}{g(z)}\left(\frac{q(z)}{q^{\prime}(z)}+\frac{v z}{\mu q(z)}\right)\right]\right)\right\} .
$$

If $p(z) \neq 0, z \in U$ satisfies the differential subordination

$$
g(z)\left[\mu p(z)+v \frac{z p^{\prime}(z)}{p(z)}\right]<g(z)\left[\mu q(z)+v \frac{z q^{\prime}(z)}{q(z)}\right],
$$

then $p<q$ and $q$ is the best dominant.

Proof. Define the functions $\theta$ and $\phi$ as follows:

$$
\theta(w(z)):=\mu w(z) g(z) \quad \text { and } \quad \phi(w(z)):=\frac{v g(z)}{w(z)} .
$$

Obviously, the functions $\theta$ and $\phi$ are analytic in domain $D=\mathbb{C} \backslash\{0\}$ and $\phi(w) \neq 0$ in $D$. Now, define the functions $Q$ and $h$ as follows:

$$
\begin{gathered}
Q(z):=z q^{\prime}(z) \phi(q(z))=v g(z) \frac{z q^{\prime}(z)}{q(z)}, \\
h(z):=\theta(q(z))+Q(z)=\mu q(z) g(z)+v g(z) \frac{z q^{\prime}(z)}{q(z)} .
\end{gathered}
$$

Then in view of condition (9), we obtain $Q$ is starlike in $U$ and $\Re\left\{\frac{z h^{\prime}(z)}{Q(z)}\right\}>0$ for $z \in U$. Furthermore, in view of condition (10) we have

$$
\theta(p(z))+z p^{\prime}(z) \phi(p(z)) \prec \theta(q(z))+z q^{\prime}(z) \phi(q(z)) .
$$

Therefore, the proof follows from Lemma 2.

By letting $\mu=1, v=\alpha, g(z):=\frac{z f^{\prime}(z)}{\Phi_{1}(z)}$ and $p=\frac{z f^{\prime}(z)}{f(z)}$ in Theorem 2 we have

Corollary 4. Let $q, q(z) \neq 0$, be a univalent function in $U$, and $g(z) \neq 0$ be analytic in $U$ satisfy (9). If $\frac{z f^{\prime}(z)}{f(z)} \neq 0, z \in U$ and

$$
\frac{z f^{\prime}(z)}{\Phi_{1}(z)}\left[(1-\alpha) \frac{z f^{\prime}(z)}{f(z)}+\alpha\left(1+\frac{z f^{\prime \prime}(z)}{f^{\prime}(z)}\right)\right]<\frac{z f^{\prime}(z)}{\Phi_{1}(z)}\left[q(z)+\alpha \frac{z q^{\prime}(z)}{q(z)}\right]
$$

then $\frac{z f^{\prime}(z)}{f(z)}<q$ and $q$ is the best dominant. 
By setting $\mu=1-\alpha, v=\alpha, g(z):=1$ and $p=\frac{z f^{\prime}(z)}{f(z)}$ in Theorem 2 we obtain the following result which can be found in [5, Theorem 3.2].

Corollary 5. Let $q, q(z) \neq 0$, be a univalent function in $U$, and $g(z) \neq 0$ be analytic in $U$ satisfy (9). If $\frac{z f^{\prime}(z)}{f(z)} \neq 0, z \in U$ and

$$
(1-2 \alpha) \frac{z f^{\prime}(z)}{f(z)}+\alpha\left(1+\frac{z f^{\prime \prime}(z)}{f^{\prime}(z)}\right)<(1-\alpha) q(z)+\alpha \frac{z q^{\prime}(z)}{q(z)}
$$

then $\frac{z f^{\prime}(z)}{f(z)}<q$ and $q$ is the best dominant.

By assuming $\mu=1-\alpha, v=\alpha, g(z):=1$ and $p(z)=\frac{z f^{\prime}(z)}{\Phi(f(z))}$ in Theorem 2 we obtain the following result which can be found in [5, Theorem 3.3].

Corollary 6. Let $q, q(z) \neq 0$, be a univalent function in $U$, and $g(z) \neq 0$ be analytic in $U$ satisfy (9). If $\frac{z f^{\prime}(z)}{\Phi(f(z))} \neq 0, z \in U$ and

$$
(1-\alpha) \frac{z f^{\prime}(z)}{\Phi(f(z))}+\alpha\left(1+\frac{z f^{\prime \prime}(z)}{f^{\prime}(z)}-\frac{z \Phi(f(z))}{\Phi^{\prime}(f(z))}\right) \prec(1-\alpha) q(z)+\alpha \frac{z q^{\prime}(z)}{q(z)},
$$

then $\frac{z f^{\prime}(z)}{\Phi(f(z))}<q$ and $q$ is the best dominant.

Finally, by assuming $\mu=1-\alpha, v=\alpha, g(z):=\frac{z f^{\prime}(z)}{\Phi_{1} f((z))}$ and $p(z)=\frac{z f^{\prime}(z)}{\Phi_{2}(f(z))}$ in Theorem 2 we obtain the following result:

Corollary 7. Let $q, q(z) \neq 0$, be a univalent function in $U$, and $g(z) \neq 0$ be analytic in $U$ satisfy (9). If $\frac{z f^{\prime}(z)}{\Phi_{2}(f(z))} \neq 0, z \in U$ and

$$
\frac{z f^{\prime}(z)}{\Phi_{1} f((z))}\left[(1-\alpha) \frac{z f^{\prime}(z)}{\Phi_{2}(f(z))}+\alpha\left(1+\frac{z f^{\prime \prime}(z)}{f^{\prime}(z)}-\frac{z \Phi_{2}^{\prime}(f(z))}{\Phi_{2}(f(z))}\right)\right] \prec \frac{z f^{\prime}(z)}{\Phi_{1}(z)}\left[(1-\alpha) q(z)+\alpha \frac{z q^{\prime}(z)}{q(z)}\right]
$$

then $\frac{z f^{\prime}(z)}{\Phi_{2}(f(z))}<q$ and $q$ is the best dominant.

\section{References}

[1] L. Brickman, $\Phi$-like analytic functions, I, Bull. Amer. Math. Soc., 79(1973), 555-558.

[2] St. Ruscheweyh, A subordination theorem for $\Phi$-like functions, J. London Math. Soc., 2(1976), 275-280.

[3] S. Singh, S. Gupta and S. Singh, On a problem of univalence of functions satisfying a differential inequality, Mathematical Inequalities and Applications, 10(2007), 95-98.

[4] V. Singh, S. Singh and S. Gupta, A problem in the theory of univalent functions, Integral Transforms and Special Functions, 16(2005), 179-186. 
[5] S. Singh, S. Gupta and S. Singh, An extension of the region of variability of a subclass of univalent functions, J. Inequal. Pure and Appl. Math., 10(2009), Art. 113, 7 pp.

[6] Z. Lewandowski, S. S. Miller and E. Zlotkiewicz, Generating functions for classes of uni-valent functions, Proc. Amer. Math. Soc., 56(1976), 111-117.

[7] J.-L. Li and S. Owa, Sufficient conditions for starlikeness, Indian J. Pure Appl. Math., 33(2002), 313-318.

[8] C. Ramesha, S. Kumar and K. S. Padmanabhan, A sufficient condition for starlikeness, Chinese J. Math., 23(1995), 167-171.

[9] Oh Sang Kwon, Sufficient conditions for starlikeness and strongly-starlikeness, Commun. Korean Math. Soc., 22(2007), 19-26.

[10] V. Ravichandran, C. Selvaraj and R. Rajalakshmi, Sufficient conditions for starlike functions of order $\alpha$, J. Inequal. Pure and Appl. Math., 3(2002), 1-6.(Art.81).

[11] K. S. Padmanabhan, On sufficient conditions for starlikeness, Indian J. Pure Appl. Math., 32(2001), 543-550.

[12] M. Obradovic and S. B. Joshi, On certain classes of strongly starlike functions, Taiwanese J. Math., 2(1998), 297-302.

[13] M. Obradovic, S. B. Joshi and I. Jovanovic, On certain sufficient conditions for starlike- ness and convexity, Indian J. Pure Appl. Math., 29(1998), 271-275.

[14] S. Singh and S. Gupta, First order differential subordination and starlikeness of ana- lytic maps in unit disc, Kyungpook Math.J. 45(2005), 395-404.

[15] S. Singh and S. Gupta, A differential subordination and starlikeness of analytic func- tions, Appl. Math. lett., 19(2006), 618-627.

[16] N. Xu and D.-G. Yang, Some criteria for starlikeness and strongly starlikeness, Bull. Korean Math. Soc., 42 (2005), 579-590.

[17] I. S. Jack, Functions starlike and convex of order K, J. London Math. Soc., 3(1971), 469-474 .

[18] S. S. Miller and P. T. Mocanu, Differential Subordinantions: Theory and Applications. Pure and Applied Mathematics No.225 Dekker, New York, 2000.

Institute of Mathematical Sciences, University Malaya, 50603, Kuala Lumpur, Malaysia.

E-mail: rabhaibrahim@yahoo.com 